\title{
Elderly Subset Analysis of Randomized Phase III Study Comparing Pemetrexed Plus Carboplatin with Docetaxel Plus Carboplatin as First-Line Treatment for Patients with Locally Advanced or Metastatic Non-Small Cell Lung Cancer
}

\author{
José Rodrigues Pereira $\cdot$ Rebecca Cheng $\cdot$ \\ Mauro Orlando $\cdot$ Joo-Hang Kim $\cdot$ Helen Barraclough
}

Published online: 26 November 2013

(C) The Author(s) 2013. This article is published with open access at Springerlink.com

\begin{abstract}
Background Many physicians consider platinum-doublet chemotherapy inappropriate for elderly patients, regardless of their medical fitness.

Objective This was a retrospective subgroup analysis of data from a multicenter, randomized, phase III clinical trial evaluating pemetrexed + carboplatin versus docetaxel + carboplatin in elderly chemo-naive patients with advanced, nonsquamous non-small cell lung cancer (NSCLC).

Methods Data from elderly patients (aged $\geq 65$ years and $\geq 70$ years) were evaluated using the same statistical methods as those used in patients aged $<70$ years and qualified intent-to-treat (Q-ITT) populations. The primary objective of the clinical trial was comparison of pemetrexed + carboplatin with docetaxel + carboplatin in terms of survival without grade 3 or 4 toxicity in chemo-naive NSCLC patients.
\end{abstract}

Clinicaltrials.gov NCT00520676.

J. R. Pereira

Instituto Brasileiro Cancerologia Toracica, Sao Paulo, Brazil

R. Cheng

Eli Lilly and Company, Taipei, Taiwan

M. Orlando

Eli Lilly Interamerica, Buenos Aires, Argentina

J.-H. Kim

Yonsei Cancer Center, Yonsei Univ. Health System,

Seoul, Korea

H. Barraclough $(\bowtie)$

Eli Lilly Australia, 112 Wharf Road, West Ryde,

NSW 2114, Australia

e-mail: barraclough_helen@lilly.com
Results The $\geq 65$ - and $\geq 70$-year age groups had 68 and 37 patients, respectively. Among patients aged $\geq 65$ years, the adjusted hazard ratio (HR) for survival without grade 3-4 toxicity (HR 0.40, $95 \%$ confidence interval [CI] $0.23-0.70$ ) favored pemetrexed + carboplatin; this was similar to the HRs in patients aged $\geq 70$ years (HR 0.43 , $95 \%$ CI $0.20-0.92$ ), patients aged $<70$ years (HR 0.44, $95 \%$ CI $0.32-0.62$ ), and the Q-ITT population (HR 0.45, $95 \%$ CI $0.34-0.61)$. The median values for overall survival (OS) and progression-free survival (PFS) were similar across all age-group subsets and the Q-ITT population. The HRs for OS and PFS were similar for all age-group subsets, except for the $\geq 70$-year age group, which favored pemetrexed + carboplatin to a greater extent. The toxicity profile was similar across age groups, with the exception of diarrhea, mucosal inflammation, and grade 3-4 neutropenia and leukopenia, which were slightly more common in elderly patients in both treatment arms. Between-arm differences in the toxicity profiles for the $\geq 65-, \geq 70$ - and $<70$-year age subgroups were similar to those in the Q-ITT population. There were no on-study deaths or unexpected toxicities.

Conclusion The benefits of pemetrexed + carboplatin were maintained, and toxicity was manageable in both elderly subgroups. The favorable risk-benefit profile of pemetrexed + carboplatin makes it an appropriate firstline treatment option for elderly patients with advanced nonsquamous NSCLC.

\section{Introduction}

Lung cancer predominantly affects the elderly; the median age of patients with non-small cell lung cancer (NSCLC) is 71 years [1]. Platinum-based doublets are the cornerstone 
of treatment for advanced NSCLC patients with a good performance status. Although these produce a survival benefit in elderly patients, only $30 \%$ receive this treatment, often because of physician concerns regarding anticipated age-related toxicity.

To mitigate toxicity, alternative agents have been incorporated into platinum-based backbones. Pemetrexed has been incorporated into first-line doublets [2-4], and carboplatin has been used instead of cisplatin [5, 6]. In a phase III trial, pemetrexed + carboplatin had a more favorable risk-benefit ratio than docetaxel + carboplatin [2]. This exploratory analysis evaluated the efficacy and safety of pemetrexed + carboplatin in elderly patients.

\section{Patient and Methods}

This was a retrospective subset analysis of a phase III trial comparing pemetrexed + carboplatin and docetaxel + carboplatin as first-line treatment in advanced nonsquamous NSCLC [2]. Data from elderly patients were evaluated in separate analyses (of patients aged $\geq 65$ and $\geq 70$ years) from the analyses of 20 - to $<70$-year-old patients (i.e. patients aged $<70$ years).

Patients were administered the study drugs in an intravenous infusion on day 1 of each 21-day cycle, up to a maximum of six cycles. Pemetrexed $\left(500 \mathrm{mg} / \mathrm{m}^{2}\right)$ or docetaxel $\left(75 \mathrm{mg} / \mathrm{m}^{2}\right.$ ), and carboplatin (area under the curve: $5 \mathrm{mg} / \mathrm{mL} \times \min$ ) were administered. Patients in the pemetrexed + carboplatin group were supplemented with at least five daily doses of oral folic acid (350-1,000 $\mu \mathrm{g}$ once daily) within 7 days of the first dose of pemetrexed and were required to take daily folic acid supplements for 21 days following treatment; an intramuscular injection of vitamin $\mathrm{B}_{12}(1,000 \mu \mathrm{g})$ was given within 7 days of the first dose of pemetrexed and once every three cycles thereafter; and oral dexamethasone (4 mg twice daily) was required the day before, the day of, and the day after administration of pemetrexed [2]. Patients in the docetaxel + carboplatin group received supplementation with oral dexamethasone ( $8 \mathrm{mg}$ twice daily) the day before, the day of, and the day after administration of docetaxel.

Time-to-event endpoints were analyzed using Cox proportional hazard models adjusted for Eastern Cooperative Oncology Group (ECOG) performance status ( 0 or 1 versus 2), disease stage (IIIB versus IV), ethnicity (East Asian versus others), gender (male versus female), and smoking status (never versus ever). The between-arm tumor response and disease control rates were compared using multivariate logistic regression models adjusted for the same covariates. Toxicities were compared using Fisher's exact text.

\section{Results}

\subsection{Study Population}

The $<70$-, $\geq 65$-, and $\geq 70$-year age groups had 174,68 , and 37 patients, respectively, with median ages of 57.5, 70.3, and 73.1 years, respectively. Between-arm imbalances in the $<70-, \geq 65-$, and $\geq 70$-year age groups favored the docetaxel + carboplatin arm among women (pemetrexed + carboplatin $39.3,28.6$, and $41.2 \%$, respectively, versus docetaxel + carboplatin 51.8, 51.5, and $55.0 \%$, respectively) and never smokers (pemetrexed + carboplatin $34.8,14.3$, and $17.6 \%$, respectively, versus docetaxel + carboplatin $38.8,33.3$, and $40.0 \%$, respectively) [Table 1].

\subsubsection{Treatment Delivery}

The six-cycle completion rates in the $<70-, \geq 65-$, and $\geq 70$ year age groups were as follows: pemetrexed + carboplatin 58.4, 57.1, and 52.9\%, respectively; docetaxel + carboplatin 44.7, 54.5, and $60.0 \%$, respectively. Among the qualified intent-to-treat (Q-ITT) population, the six-cycle completion rates were pemetrexed + carboplatin $57.5 \%$ and docetaxel + carboplatin $47.6 \%$ [2]. Dose reductions in the $<70-, \geq 65$-, and $\geq 70$-year age groups were experienced by $9.0,2.9$, and $5.9 \%$ of pemetrexed + carboplatin-treated patients, respectively, and by $23.5,39.4$, and $40.0 \%$ of docetaxel + carboplatin-treated patients, respectively; in the Q-ITT population, dose reductions were experienced by $8.5 \%$ of pemetrexed + carboplatin-treated patients and by $26.7 \%$ of docetaxel + carboplatin-treated patients [2]. All dose reductions were due to adverse events (AEs). The median relative dose intensities for pemetrexed + carboplatin in the $<70$-, $\geq 65$-, and $\geq 70$-year age groups were as follows: pemetrexed 95.0, 95.4, and 95.5\%, respectively; carboplatin 92.4, 94.6, and $94.1 \%$, respectively; and those for docetaxel + carboplatin were as follows: docetaxel 96.4, 89.7 , and $89.1 \%$, respectively; carboplatin $89.9,84.4$, and $82.9 \%$, respectively. Among Q-ITT patients, the median relative dose intensities for pemetrexed + carboplatin were $95.3 \%$ for pemetrexed and $92.7 \%$ for carboplatin, and those for docetaxel + carboplatin were $95.0 \%$ for docetaxel and $88.7 \%$ for carboplatin [2].

\subsection{Survival Without Toxicity}

Survival without grade 3 or 4 toxicity was significantly improved in pemetrexed + carboplatin-treated patients in all age groups (Table 2). The adjusted hazard ratio (HR) for the <70-year age group (median 3.4 months for pemetrexed + carboplatin versus 0.7 months for 


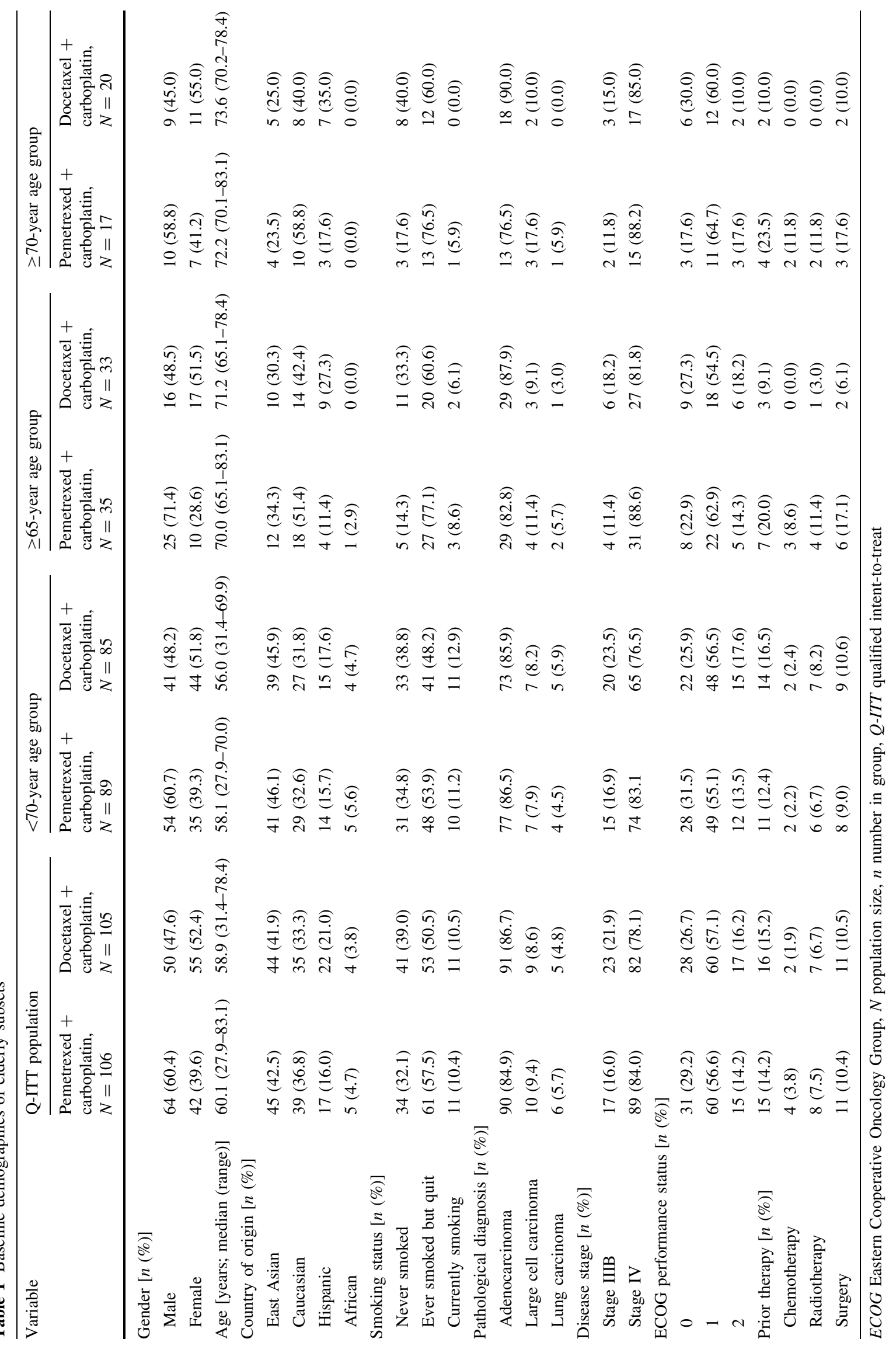




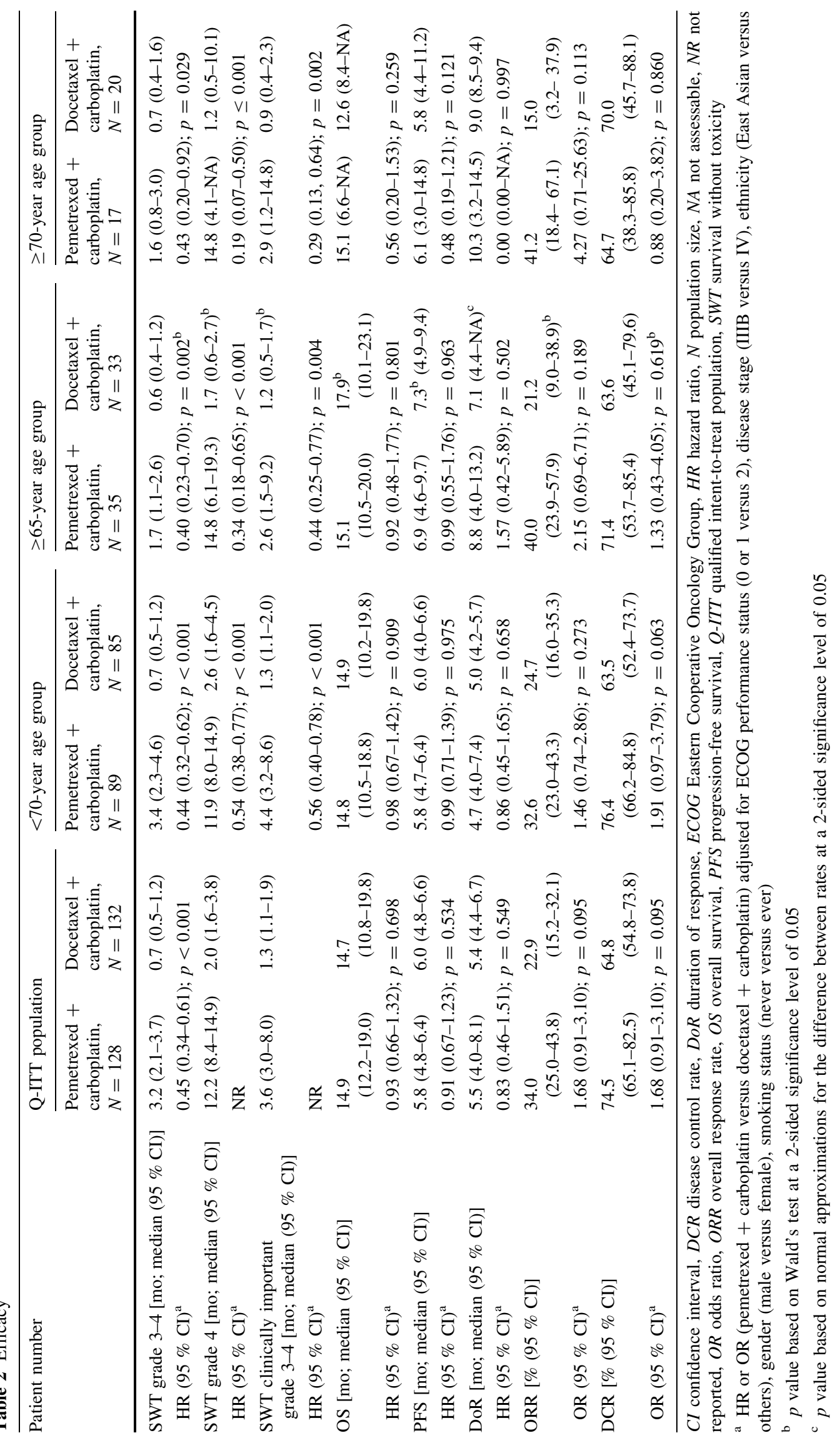


docetaxel + carboplatin; adjusted HR $0.44,95 \%$ confidence interval [CI] $0.32-0.62 ; p<0.001)$ was consistent with those in the $\geq 65$-year age group (median 1.7 months for pemetrexed + carboplatin versus 0.6 months for docetaxel + carboplatin; adjusted HR $0.40,95 \%$ CI $0.23-0.70 ; p=0.002$ ), the $\geq 70$-year age group (median 1.6 months for pemetrexed + carboplatin versus 0.7 months for docetaxel + carboplatin; adjusted HR $0.43,95 \%$ CI $0.20-0.92 ; p=0.029$ ) [Table 2] and the Q-ITT population [2]. Survival without grade 4 toxicity and survival without clinically important grade 3 or 4 toxicity were also significantly improved in the pemetrexed + carboplatin treatment arm for all age subgroups (Table 2). The magnitude of the HR change favoring pemetrexed + carboplatin was greater for the $\geq 70$-year age group than for the $<70$-year age group with respect to survival without grade 4 toxicity and survival without clinically important grade 3 or 4 toxicity (Table 2 ).

\subsection{Efficacy}

Among elderly patients, there were no statistically significant between-treatment group differences in overall survival (OS) or progression-free survival (PFS) [Table 2]. The median values were similar across all age-group subsets. The HRs were similar for all age-group subsets except for the $\geq 70$-year age group, which favored pemetrexed + carboplatin to a greater extent (Table 2). The tumor response rate favored the pemetrexed + carboplatin arm across all age groups and, similarly to OS and PFS, the magnitude of benefit increased for the elderly subsets (Table 2).

\subsection{Safety}

Fewer PCb-treated patients experienced $\geq 1$ drug-related Grade 3 or 4 treatment-emergent adverse event (TEAE) than DCb-treated patients $(\geq 65 / \geq 70) \quad(\mathrm{PCb}, 54.3 \% /$ $58.8 \%$; DCb, $81.8 \% / 85.0 \% ; \mathrm{p}=0.020 / \mathrm{p}=0.136)$, but the proportions of patients experiencing $\geq 1$ drug-related TEAE of any grade were similar $(<70 / \geq 65 / \geq 70)$ : $(\mathrm{PCb}$, $79.8 \% / 88.6 \% / 82.4 \%$; $\quad$ DCb, $90.6 \% / 87.9 \% / 90.0 \%$; $\mathrm{p}=0.056 / \mathrm{p}=1.000 / \mathrm{p}=0.644)$. Discontinuations due to possibly drug-related serious AEs occurred in two $\geq 65$ year-old patients in each arm (pemetrexed + carboplatin: 1 anemia and 1 decreased platelet count; docetaxel + carboplatin: 2 febrile neutropenia) and in one $\geq 70$-year-old patient in each arm (pemetrexed + carboplatin: anemia; docetaxel + carboplatin: febrile neutropenia). Notably, there were no on-therapy deaths in either treatment arm in elderly patients, patients aged $<70$ years, or the Q-ITT population.
In patients aged $\geq 65$ years, there were significantly lower incidences of all-grade drug-related neutropenia, leukopenia, febrile neutropenia, alopecia, and diarrhea in the pemetrexed + carboplatin arm than in the docetaxel + carboplatin arm (Table 3). Docetaxel + carboplatin-treated patients aged $\geq 65$ years may be more likely to suffer febrile neutropenia than the docetaxel + carboplatin-treated Q-ITT population. Additionally, in patients aged $\geq 65$ years, the incidences of grade 3 or 4 neutropenia, leukopenia, and febrile neutropenia were significantly lower in the pemetrexed + carboplatin arm.

In general, the between-arm trends and incidences of possibly drug-related treatment-emergent AEs were similar in patients aged $\geq 65$ years and the Q-ITT population. There were some differences within the $\geq 70$-year age group, perhaps because of the small sample size. Nonetheless, regardless of treatment, elderly patients may be more likely to suffer diarrhea, constipation, abdominal pain (except in docetaxel + carboplatin-treated patients $\geq 70$ years), and mucosal inflammation than patients aged $<70$ years and the Q-ITT population (Table 3).

A summary of dose reductions due to AEs in the safety population age-group subsets ( $<70$ years, $\geq 65$ years, and $\geq 70$ years) demonstrated that significantly more patients in the docetaxel + carboplatin arm than in the pemetrexed + carboplatin arm had at least one dose reduction due to AEs: pemetrexed + carboplatin 9.0, 2.9, and $5.9 \%$, respectively; docetaxel + carboplatin 23.5, 39.4, and $40.0 \%$, respectively; $p=0.013,0.001$, and 0.023 , respectively). Notably, this difference was driven predominantly by neutropenia in the docetaxel + carboplatin arm, which led to at least one dose reduction due to an $\mathrm{AE}$ significantly more often in each of the age-group subsets: pemetrexed + carboplatin 2.2, 0.0 , and $0.0 \%$, respectively; docetaxel + carboplatin 17.6, 24.2, and $25.0 \%$, respectively; $p<0.001,0.002$, and 0.050 , respectively).

\subsection{Post-Discontinuation Anti-Cancer Therapy}

Within the $<70-, \geq 65$-, and $\geq 70$-year age-group subsets, $62.9,40.0$, and $17.6 \%$ of pemetrexed + carboplatin-treated patients, respectively, and $48.2,48.5$, and $55.0 \%$ of docetaxel + carboplatin-treated patients, respectively, received post-study therapy. Among the Q-ITT patients, $55.7 \%$ of pemetrexed + carboplatin-treated patients and $49.5 \%$ of docetaxel + carboplatin-treated patients received post-discontinuation therapy [2]. Within the $<70$-, $\geq 65$-, and $\geq 70$-year age-group subsets, the most common post-discontinuation chemotherapeutic agent used in pemetrexed + carboplatin-treated patients was docetaxel (used in 23.6, 11.4, and 5.9\%, respectively), and in docetaxel + carboplatin-treated patients it was pemetrexed (14.1, 12.1, and $15.0 \%$, respectively). Within the $<70-$, 


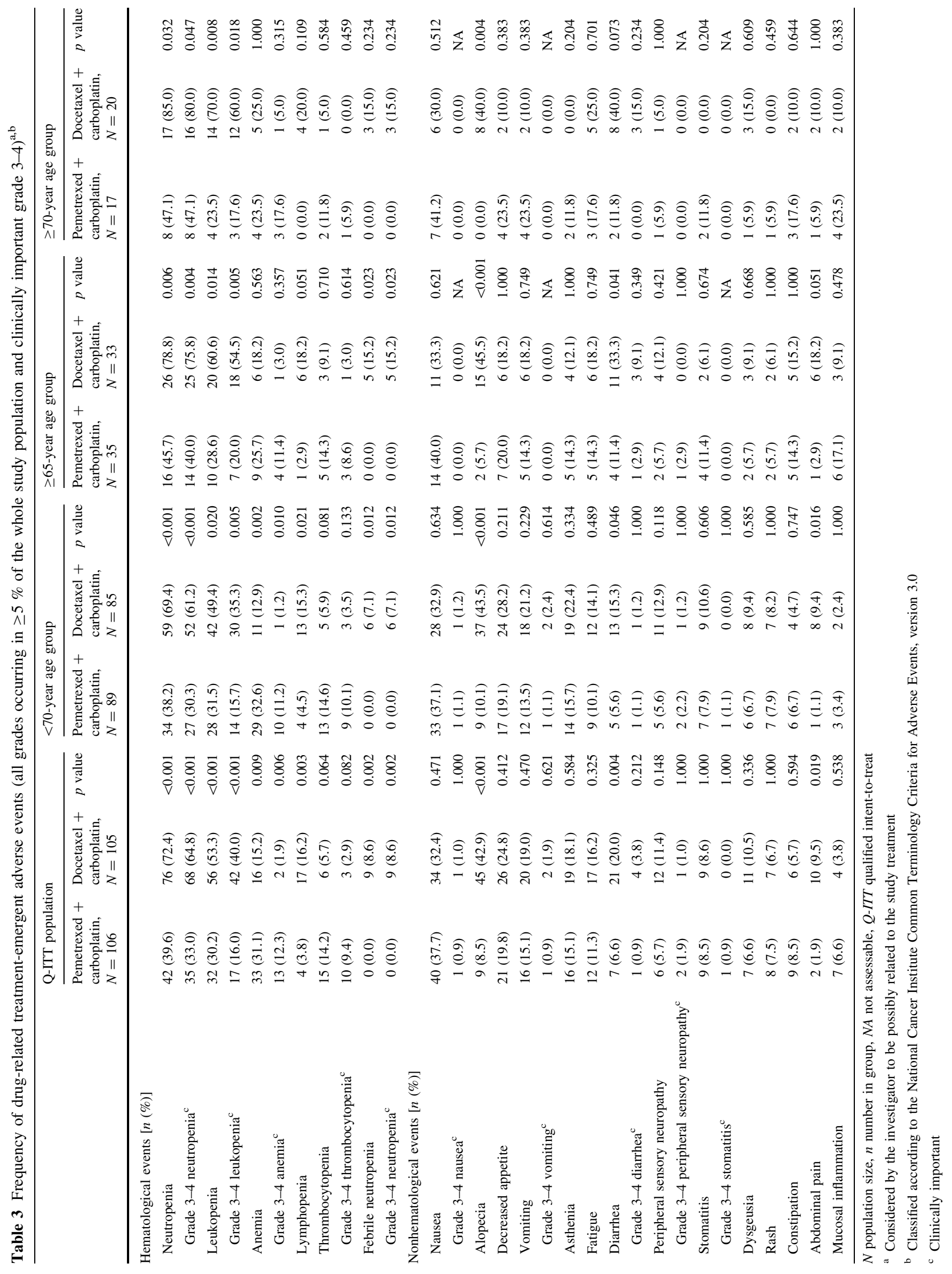


$\geq 65$-, and $\geq 70$-year age-group subsets, post-study epidermal growth factor receptor tyrosine kinase inhibitors were received by $22.5,14.3$, and $11.8 \%$ of pemetrexed + carboplatin-treated patients, respectively, and by $28.2,27.3$, and $20.0 \%$ of docetaxel + carboplatin-treated patients, respectively. Post-study radiotherapy was received by 21.3 , 8.6, and $0.0 \%$ of pemetrexed + carboplatin-treated patients, respectively, and by $22.4,21.2$, and $25.0 \%$ of docetaxel + carboplatin-treated patients, respectively.

\section{Discussion and Conclusion}

Retrospective studies suggest that elderly patients can receive a clinical benefit from platinum-based chemotherapy similar to that seen in younger patients; toxicity may be increased in this population but is still generally acceptable. Nevertheless, physicians still hesitate to use these regimens in elderly patients [7, 8]. Mortality rates for elderly patients with lung cancer have increased over the decades [9]. This could be partly related to lower chemotherapy usage in the elderly [10].

We performed a retrospective analysis of elderly patient subsets (aged $\geq 65$ and $\geq 70$ years) within a phase III trial evaluating pemetrexed + carboplatin and docetaxel + carboplatin in advanced nonsquamous NSCLC [2]. Elderly patients treated with pemetrexed + carboplatin tolerated slightly higher dose intensities and experienced fewer dose reductions than elderly patients treated with docetaxel + carboplatin. The benefits of pemetrexed + carboplatin were maintained in elderly patients with advanced NSCLC. As seen in the Q-ITT population and the $<70$-year age group, elderly pemetrexed + carboplatin-treated patients experienced longer survival without toxicity than docetaxel + carboplatin-treated patients did. There were no statistically significant between-treatment arm differences in OS, PFS, or the response rate among elderly patients, among patients aged $<70$ years, and in the Q-ITT population; however, the response rate was numerically higher in pemetrexed + carboplatin-treated patients than in docetaxel + carboplatin-treated patients, and the between-arm response differences appeared greater in elderly patients than in the those aged $<70$ years and the Q-ITT population. This might be a reflection of greater variability due to the smaller number of patients in the $\geq 70$-year age group.

The retention of pemetrexed + carboplatin-related benefits in elderly patients is likely due to this regimen's favorable AE profile. Elderly patients treated with pemetrexed + carboplatin experienced lower rates of most hematological AEs (i.e., neutropenia, leukopenia, lymphopenia, febrile neutropenia) than elderly patients treated with docetaxel + carboplatin. Moreover, there were reduced rates of alopecia and diarrhea among elderly patients treated with pemetrexed + carboplatin. In both arms, the AE trends in the elderly mostly mirrored those of the Q-ITT population and the <70-year age group. Importantly, there were no unexpected AEs in either treatment arm, nor were there on-study deaths among elderly patients. The between-arm toxicity profile difference was consistent across all age-group subsets. There was a slight increase in selected toxicities (mucosal inflammation, diarrhea, neutropenia, and leukopenia) in the elderly age groups compared with the $<70$-year age-group subset, regardless of the treatment arm. This may have contributed to the improved survival without grade 4 toxicity and survival without grade 3 or 4 clinically important toxicity differences observed with respect to the magnitude of the HR in favor of pemetrexed + carboplatin.

Subset analyses of pemetrexed registration trials showed that the benefit of pemetrexed is maintained in elderly advanced NSCLC patients without compromising tolerability $[11,12]$. In elderly first-line NSCLC patients treated with pemetrexed + cisplatin, the rates of neutropenia, thrombocytopenia, and febrile neutropenia appeared to increase with age [11]. However, in all age groups, the $<70$-year age group, the $\geq 65$-year age group, and $\geq 70$ year age group in our trial, the rates of neutropenia (39.6, $38.2,45.7$, and $47.1 \%$, respectively), thrombocytopenia (14.2, 14.6, 14.3, and $11.8 \%$, respectively), and febrile neutropenia $(0,0,0$, and $0 \%$, respectively) were approximately the same in pemetrexed-treated patients. Differences between trials could possibly be attributed to the use of carboplatin; however, this seems unlikely because carboplatin is associated with lower rates of nausea, vomiting, and nephrotoxicity, but a higher rate of thrombocytopenia, relative to cisplatin $[5,6]$.

In this exploratory analysis, defining $\geq 65$ years as 'elderly' allowed for sufficient patient numbers to be included in the main subgroup. Further analysis of $\geq 70$ year-old patients showed efficacy and safety similar to those in $\geq 65$-year-old patients, but the former was limited by a small population size, yielding more variable results. Our study underscores that NSCLC patients, regardless of age, benefit from appropriate treatment [13], and supports the idea that treatment selection in the elderly should not be based solely on chronological age.

This exploratory analysis suggests that the outcomes of elderly patients with nonsquamous NSCLC are consistent with those in the $<70$-year age group and the Q-ITT population with respect to dose intensity, efficacy, and tolerability. Therefore, with few limitations, elderly patients with advanced nonsquamous NSCLC and good performance status should be treated similarly to younger patients. We and others have shown that platinum-based doublet therapy is a tolerable, viable option for elderly 
advanced NSCLC patients [11, 12, 14]. However, our conclusions are hypothesis generating, as this retrospective analysis had a small sample size and unbalanced betweenarm patient characteristics. The limitations of retrospective elderly patient studies include potential differences between chronological age and medical fitness, elderly population heterogeneity, arbitrary age cut-offs, and ageassociated co-morbidities. Our selection criteria of fit elderly patients may not have been applicable to the general elderly population. Therefore, a prospective clinical trial involving a carefully controlled group of elderly patients is warranted.

Acknowledgments This work was supported by Eli Lilly and Company. The sponsor was responsible for the design and conduct of the trial, as well as the collection, analysis, and interpretation of data. The manuscript was prepared with input from all authors; all authors approved the final version for submission to the journal.

Rebecca Cheng and Mauro Orlando are employees of Eli Lilly and Company and own stock in the company. Helen Barraclough is an employee of Eli Lilly and Company. Joo-Hang Kim's institution received a grant from Eli Lilly and Company for this clinical trial. José Rodrigues-Pereira has no relevant conflicts of interest to report.

The authors wish to thank the patients, their families, and the study personnel who participated in this clinical trial. We also thank Shu Bin Liu and Wei Shan Shi for assistance with statistical analyses. Medical writing support was provided by Lori Kornberg and Emily Cullinan, who are full-time employees of inVentiv Health Clinical.

Open Access This article is distributed under the terms of the Creative Commons Attribution Noncommercial License which permits any noncommercial use, distribution, and reproduction in any medium, provided the original author(s) and the source are credited.

\section{References}

1. Langer CJ. Clinical evidence on the undertreatment of older and poor performance patients who have advanced non-small-cell lung cancer: is there a role for targeted therapy in these cohorts? Clin Lung Cancer. 2011;12(5):272-9.

2. Rodrigues-Pereira J, Kim JH, Magallanes M, et al. A randomized phase 3 trial comparing pemetrexed/carboplatin and docetaxel/ carboplatin as first-line treatment for advanced, nonsquamous non-small cell lung cancer. J Thorac Oncol. 2011;6(11):1907-14.
3. Scagliotti GV, Parikh P, von Pawel J, et al. Phase III study comparing cisplatin plus gemcitabine with cisplatin plus pemetrexed in chemotherapy-naive patients with advanced-stage non-small-cell lung cancer. J Clin Oncol. 2008;26(21):3543-51.

4. Li M, Zhang Q, Fu P, et al. Pemetrexed plus platinum as the firstline treatment option for advanced non-small cell lung cancer: a meta-analysis of randomized controlled trials. PLoS One. 2012; 7(5):e37229.

5. Ardizzoni A, Boni L, Tiseo M, et al. Cisplatin- versus carboplatin-based chemotherapy in first-line treatment of advanced non-small-cell lung cancer: an individual patient data meta-analysis. J Natl Cancer Inst. 2007;99(11):847-57.

6. Jiang J, Liang X, Zhou X, et al. A meta-analysis of randomized controlled trials comparing carboplatin-based to cisplatin-based chemotherapy in advanced non-small cell lung cancer. Lung Cancer. 2007;57(3):348-58.

7. Gridelli C, Maione P, Rossi A, et al. Treatment of advanced nonsmall-cell lung cancer in the elderly. Lung Cancer. 2009;66(3): 282-6.

8. Langer CJ, Manola J, Bernardo P, et al. Cisplatin-based therapy for elderly patients with advanced non-small-cell lung cancer: implications of Eastern Cooperative Oncology Group 5592, a randomized trial. J Natl Cancer Inst. 2002;94(3):173-81.

9. Wingo PA, Cardinez CJ, Landis SH, et al. Long-term trends in cancer mortality in the United States, 1930-1998 [published erratum appears in Cancer 2005 Jun 15;103 (12):2658]. Cancer. 2003;15(97 Suppl. 12):3133-275.

10. Ramsey SD, Howlader N, Etzioni RD, et al. Chemotherapy use, outcomes, and costs for older persons with advanced non-smallcell lung cancer: evidence from surveillance, epidemiology and end results-Medicare. J Clin Oncol. 2004;22(24):4971-8.

11. Gridelli C, Brodowicz T, Langer CJ, et al. Pemetrexed therapy in elderly patients with good performance status: analysis of two phase III trials of patients with nonsquamous non-small-cell lung cancer. Clin Lung Cancer. 2012;13(5):340-6.

12. Weiss GJ, Langer C, Rosell R, et al. Elderly patients benefit from second-line cytotoxic chemotherapy: a subset analysis of a randomized phase III trial of pemetrexed compared with docetaxel in patients with previously treated advanced non-small-cell lung cancer [published erratum appears in J Clin Oncol 2006 Dec 1;24 (34):5477]. J Clin Oncol. 2006;24(27):4405-11.

13. Davidoff AJ, Tang M, Seal B, et al. Chemotherapy and survival benefit in elderly patients with advanced non-small-cell lung cancer. J Clin Oncol. 2010;28(13):2191-7.

14. Quoix E, Zalcman G, Oster JP, et al. Carboplatin and weekly paclitaxel doublet chemotherapy compared with monotherapy in elderly patients with advanced non-small-cell lung cancer: IFCT0501 randomised, phase 3 trial. Lancet. 2011;378(9796): 1079-88. 